\title{
Effects of calcifediol versus cholecalciferol on 25(OH)D3 serum levels, appendicular muscle strength, and physical performance in post-menopausal women
}

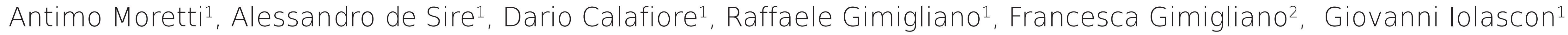

Department of Medical and Surgical Specialties and Dentistry, Second University of Naples, Naples, Italy

Department of of Physical and Mental Health and Preventive Medicine, Second University of Naples, Naples, Italy

\section{BACKGROUND}

Vitamin D can be considered a steroid hormone because of its biological effects on several tissues, beyond the bone [1]. Vitamin D stimulates the proliferation and differentiation of muscle cells through gene transcription in myoblasts, inducing an increased synthesis of muscle-specific proteins, such as myosin- and calcium-binding proteins (figure 1) [2,3]. It has been hypothesized that vitamin D supplementation increases muscle function and physical performance [4]. The aim of our study is to compare women taking cholecalciferol versus those taking calcifediol in terms of improvement of the serum levels of 25OHD3, muscle strength and muscle performance.

\section{METHODS}

In this prospective study we included postmenopausal women aged $\geq 50$ years, naïve to vitamin D or calcifediol administration. We divided this cohort into two groups: women using cholecalciferol $20 \mathrm{ug}$ (800 4 oral drops per day] and women using calcifediol $(20 \mu \mathrm{g}, 4$ oral drops per day). We evaluated at the baselin (TO) and after 6 months (T1) the following parameters: serum levels of 25(OH)D3, appendicular muscle strength, using the Hand Grip Strength Test (HGS) and the Knee Extensor Strength Test (KES), and physical performance, using the Short Physical Performance Battery (SPPB).

\section{RESULTS}

We evaluated 205 post-menopausal women, mean aged $69.28 \pm 9.16$ years, 103 treated with calcifediol and 102 with cholecalciferol. No significant differences between groups was observed in both age and BMI (table 1). Within-groups analysis demonstrated that oncedaily admistration of calcifediol significantly increased serum levels of $25(\mathrm{OH}) \mathrm{D}$, handgrip strength, knee extension strength and muscle performance compared to cholecalciferol supplementation (table 2). The same findings were confirmed in the between-groups analysis (table 3 ).

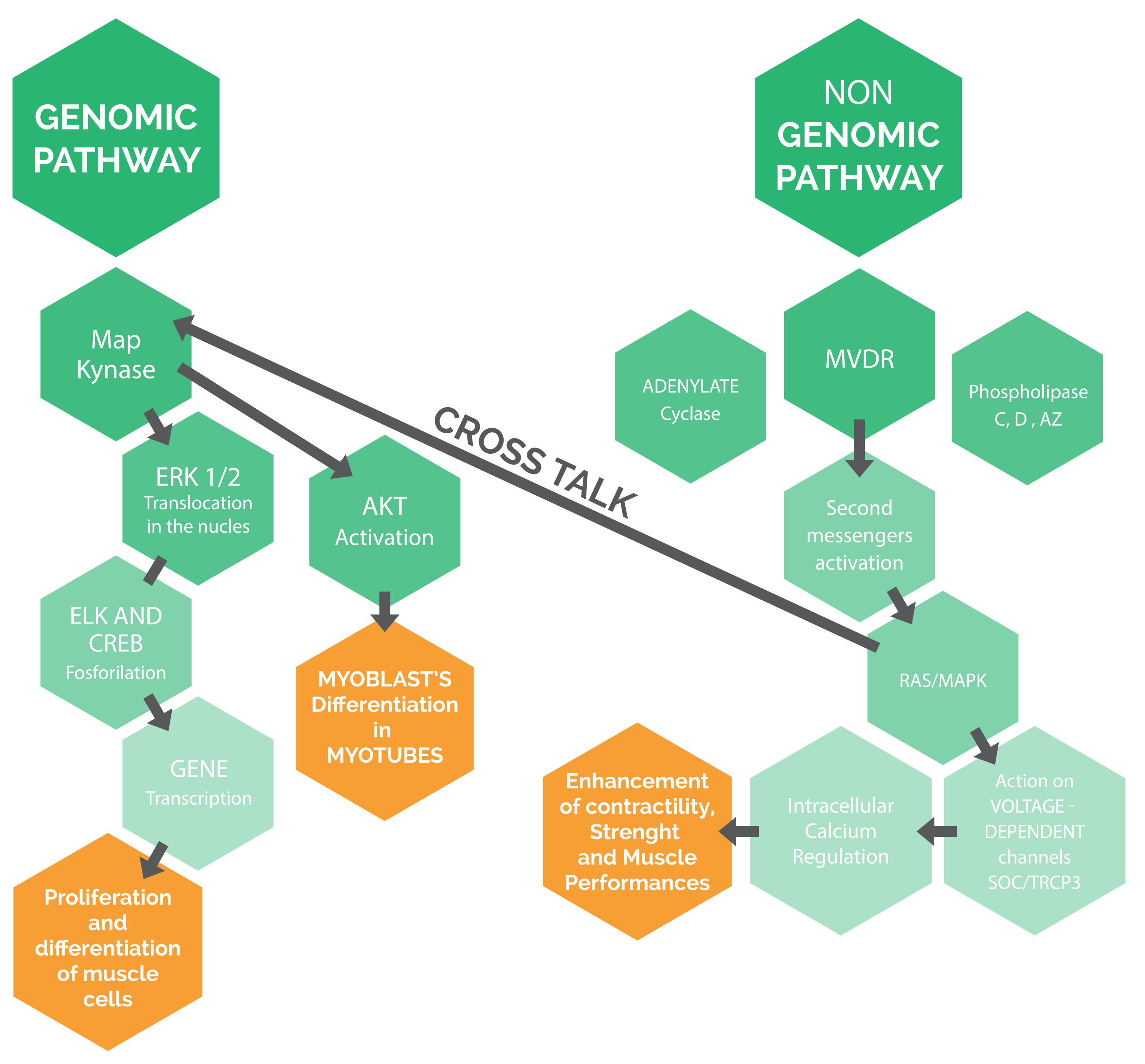

FIGURE 1: BIOLOGICAL EFFECTS OF THE VITAMIN D ON THE MUSCULAR STRENGTH AND PERFORMANCE.

\begin{tabular}{|c|c|c|c|}
\hline \multicolumn{4}{|c|}{ Table 1. Baseline characteristics } \\
\hline & Cholecalcifírol T0 ( $n=102)$ & Calcifediol TO $(n=103)$ & $P$ values \\
\hline AGE (years) & $71.19 \pm 8.13$ & $66.67 \pm 9.77$ & 0.057 \\
\hline BMI $\left(\mathrm{kg} / \mathrm{m}^{2}\right)$ & $24.75 \pm 3.23$ & $25.74 \pm 3.48$ & 0.352 \\
\hline $25(\mathrm{OH}) \mathrm{D} 3(\mathrm{ng} / \mathrm{mll})$ & $35.15 \pm 11.57$ & $31.24 \pm 13.04$ & 0.211 \\
\hline HGS(kg) & $15.36 \pm 6.06$ & $15.54 \pm 6.65$ & 0.978 \\
\hline KES $(\mathrm{kg})$ & $12.12 \pm 5.74$ & $14.49 \pm 6.92$ & 0.135 \\
\hline SPPB & $7.81 \pm 3.66$ & $8.41 \pm 3.32$ & 0.558 \\
\hline
\end{tabular}

\begin{tabular}{|c|c|c|c|}
\hline Table 2. Outcome & measurements assessed at the I & line and after 6 months of vitam & suppleme \\
\hline & Calcifediol TO (n=103) & Calcifediol T1 (n=103) & Pvalues \\
\hline $25(\mathrm{OH}) \mathrm{D} 3(\mathrm{ng} / \mathrm{mll})$ & $31.74 \pm 13.03$ & $51.80 \pm 19.85$ & $<0.001$ \\
\hline HGS(kg) & $15.54 \pm 6.65$ & $18.29 \pm 3.85$ & 0.014 \\
\hline KES(kg) & $14.49 \pm 6.92$ & $17.05 \pm 5.62$ & 0.050 \\
\hline SPPB & $8.41 \pm 3.32$ & $9.70 \pm 2.14$ & 0.008 \\
\hline & Cholecalciferol TO ( $n=102)$ & Cholecalciferol T1 ( $n=102)$ & Pvalues \\
\hline $25(\mathrm{OH}) \mathrm{D} 3(\mathrm{ng} / \mathrm{ml})$ & $35.15 \pm 11.57$ & $40.28 \pm 13.04$ & 0.118 \\
\hline HGS(kg) & $15.36 \pm 6.06$ & $16.38 \pm 5.14$ & 0.257 \\
\hline KES(kg) & $12.12 \pm 5.74$ & $12.58 \pm 5.67$ & 0.627 \\
\hline SPPB & $7.81 \pm 3.66$ & $8.02 \pm 3.41$ & 0.638 \\
\hline
\end{tabular}

\section{CONCLUSIONS}

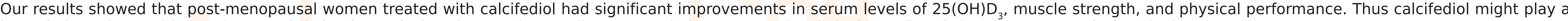
key role in patients with impairment of skeletal muscle function. Further studies are needed to support the clinical benefits of vitamin D on muscle functioning.

\begin{tabular}{|lccc|}
\hline \multicolumn{4}{|l|}{ Table 3. Outcome measurements assessed after 6 months of vitamin D supplementation. } \\
\hline & Cholecal ciferol T1 $(n=102)$ & Calcifediol T1 $(n=103)$ & P values \\
\hline $25(\mathrm{OH}) \mathrm{D} 3(\mathrm{ng} / \mathrm{ml})$ & $40.28 \pm 13.04$ & $51.80 \pm 19.85$ & 0.032 \\
\hline HGS $(\mathrm{kg})$ & $16.38 \pm 5.14$ & $18.29 \pm 3.85$ & 0.095 \\
\hline KES $(\mathrm{kg})$ & $12.58 \pm 5.67$ & $17.05 \pm 5.62$ & 0.038 \\
\hline SPPB & $8.02 \pm 3.41$ & $9.70 \pm 2.14$ & 0.019 \\
\hline (Results are expressed as means \pm standard deviations. Between groups analysis was performed using an indipendent t-test \\
\hline
\end{tabular}

IOF

\section{REFERENCES}

